\title{
Low-field extremum in the surface impedance of metals at $\mathrm{MHz}$ frequencies
}

\section{Gordon, R A}

\section{Published in:}

Physical Review B

Link to article, DOI:

10.1103/PhysRevB.22.1119

Publication date:

1980

Document Version

Publisher's PDF, also known as Version of record

Link back to DTU Orbit

Citation (APA):

Gordon, R. A. (1980). Low-field extremum in the surface impedance of metals at $\mathrm{MHz}$ frequencies. Physical Review B, 22(2), 1119-1122. https://doi.org/10.1103/PhysRevB.22.1119

\section{General rights}

Copyright and moral rights for the publications made accessible in the public portal are retained by the authors and/or other copyright owners and it is a condition of accessing publications that users recognise and abide by the legal requirements associated with these rights.

- Users may download and print one copy of any publication from the public portal for the purpose of private study or research.

- You may not further distribute the material or use it for any profit-making activity or commercial gain

- You may freely distribute the URL identifying the publication in the public portal

If you believe that this document breaches copyright please contact us providing details, and we will remove access to the work immediately and investigate your claim 


\title{
Low-field extremum in the surface impedance of metals at $\mathrm{MHz}$ frequencies
}

\author{
R. A. Gordon \\ Physics Laboratory I, The Technical University of Denmark, DK-2800 Lyngby, Denmark
}

(Received 18 May 1979)

\begin{abstract}
The first detailed measurements of the low-field extremum for a cylindrical sample at $\mathrm{MHz}$ frequencies are reported. It is shown that the model suggested by Sibbald et al. is the only interpretation of the lowfield extremum which can account either for the measurements reported here or those carried out for similar extrema observed in flat samples. The theoretical basis for this interpretation is investigated, and the importance of Fermi surface geometry is given special emphasis.
\end{abstract}

Measurements of the surface impedance of metals at liquid-helium temperatures frequently reveal a large extremum in the vicinity of zero external magnetic field at $\mathrm{MHz}$ frequencies. ${ }^{1-7}$ The temperature and frequency dependences of this low-field extremum have been measured in detail in the case of flat copper samples and, on the basis of such measurements, the surface impedance extremum has been attributed to quantized skipping electrons whose depth of penetration into the metal is much less than the skin depth. ${ }^{1}$ This interpretation of the low-field extremum has been recently contested, however, by other authors ${ }^{2}$ who argue that the measurements in copper can be interpreted equally well in terms of skipping electron trajectories which span the entire $\mathrm{MHz}$ skin depth. Other interpretations of the low-field extremum have also been suggested over the years ${ }^{3,4}$ and, in view of the fact that at least four qualitatively different inte rp retations have now been proposed to inte rpret the same or similar low-field extrema, some of them explicit to the sample geometry itself, it would be desirable to have additional detailed measurements of the low-field extremum in different samples and sample geometries which could be used to determine which of the various interpretations best describes the experimental extremum actually observed in the immediate vicinity of zero magnetic field. It is the purpose of this letter to report the results of such measurements for cylindrical samples and to give a critical discussion of how well the different interpretations can be reconciled with experiment. In particular, it is pointed out that the quantized skipping-electron interpretation is the only interpretation which has been proposed that fully agrees with detailed measurements of the low-field extremum either in flat or cylindrical samples and that the same interpretation is equally consistent with less detailed measurements of the low-field extremum in other metals.

The choice of a molybdenum cylinder represented a compromise between metals with simpler Fermi surfaces, such as the alkali and noble metals, for which the low-field extremum can be observed only with extreme difficulty, if at all, in cylindrical samples ${ }^{2}$ and metals such as tin and indium ${ }^{3,6}$ which exhibit measurable low-field extrema in cylindrical samples, but whose significantly less well-known Fermi surfaces would greatly hinder an unambiguous theoretical analysis of the phenomenon. The diameter of the sample $(4.5 \mathrm{~mm})$ and the mean free path of the skipping electrons ( $\$ 0.1 \mathrm{~mm}$ ) were such that the effects of sample curvature on the low-field extremum would be clearly observable while, at the same time, the motion of the skipping electrons around the circumference of the cylinder would be limited to a small angular region of the Fermi surface ( $\leqslant 5^{\circ}$ in arc length). ${ }^{7}$ The molybdenum cylinder was mounted inside a coaxial cylindrical coil of an rf oscillator and an external dc magnetic field applied along the common coil-sample axis. Typical experimental lowfield extrema are shown in Fig. 1. Both the line shape and position of the low-field extremum are characteristic of similar low-field extrema observed in cylindrical and flat samples of other metals.

According to the quantized-skipping-electron interpretation proposed by Sibbald et al., the experimentally observed low-field extremum in $d Z / d H$ will occur at a magnetic field $H_{0}$ such that the mean free path of the skipping electrons, $l$, is comparable to the arc length of the shortest-al lowed (i.e., lowest-quantum-number) quantizedskipping-electron trajectories whose depth of penetration into the metal is much less than the skin depth at MHz frequencies [Fig. 2(a)]; i.e., ${ }^{1}$

$$
H_{0} \cong\left(h K^{1 / 2}\right) /\left(e l^{3 / 2}\right) \text {. }
$$

Here $Z$ is the surface impedance for rf currents perpendicular to an applied dc magnetic field $H$, oriented along the sample surface, $K$ is the radius of curvature of the Fermi surface measured in the 


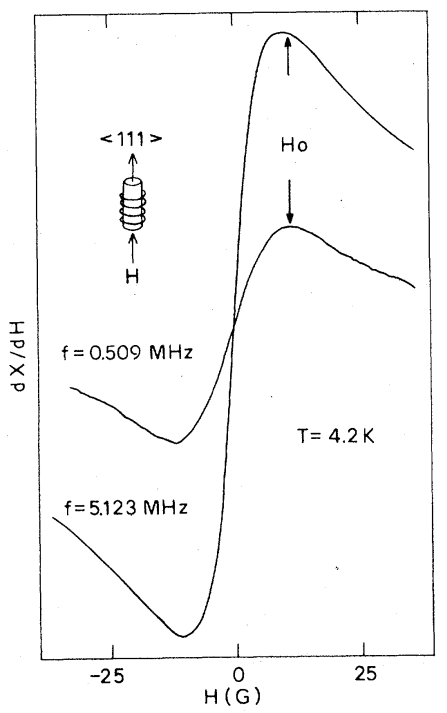

FIG. 1. Experimental derivative curves of the imaginary part of the surface impedance $d X / d H$ for a molybdenum cylinder, obtained in the same run by changing only the frequency of the external rf generator. The frequency independence of the position of the low-field extremum $H_{0}$ (at fixed temperature) illustrated above was also found to be valid up to the highest frequencies employed $(37 \mathrm{MHz})$ to within an experimental uncertainty of $\pm 5-10 \%$ in $H_{0}$.

plane of the electron motion, $h$ is Planck's constant, and $e$ is the electronic charge. ${ }^{8}$ Equation (1) predicts that the position of the low-field extremum will be frequency independent but will depend on the temperature (through the factor $l$ ) in contrast to other interpretations proposed for the low-field extremum (illustrated in Fig. 2) which predict

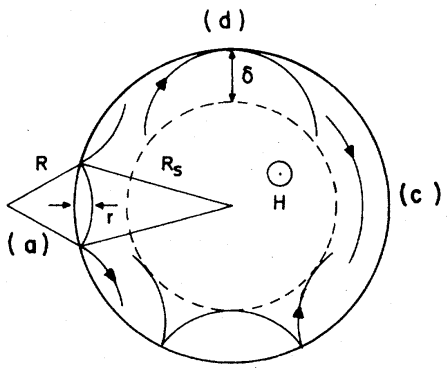

(b)

FIG. 2. Different interpretations of the low-field extremum as they would apply to a cylindrical sample: (a) the quantized skipping-electron interpretation of Sibbald et al. (Ref. 1); (b) the semiclassical skippingelectron interpretation of $\mathrm{Pal}$ and $\mathrm{Falk}$ and of Gantmakher et al. (Ref. 2); (c) the cylindrical-size-effect interpretation of Tsoi and Meirovich (Ref. 3); (d) the semiclassical skimming-electron interpretation of Drew (Ref. 4). $R$ is the cyclotron radius, $R_{s}$ is the sample radius, $\delta$ is the rf skin depth, $r$ is the depth of penetration of low-quantum-number skipping-electron trajectories $(r \ll \delta)$, and $H$ is the dc magnetic field. either a frequency-dependent value for $H_{0}$ [Figs. $2(\mathrm{~b})$ and $2(\mathrm{~d})]^{2,4}$ or a value which is temperature independent [Fig. 2(c)]. ${ }^{3}$ Detailed measurements of the low-field extremum reported here for molybdenum (Figs. 1 and 3; see also Ref. 9), as well as those previously reported for copper, ${ }^{1}$ clearly show that the position of the low-field extremum is, in fact, both independent of frequency (for changes in frequency by a factor of 10-20 or more) and very strongly temperature dependent thus ruling out all of the interpretations for the low-field extremum other than the quantized skipping-electron interpretation.

An important point which has not been explicitly emphasized before is that it is not possible to distinguish experimentally between the various skipping- and skimming-electron interpretations [Figs. 2 (a), 2 (b), and 2(d)] of the low-field extremum on the basis of the temperature dependence of the low-field extremum alone ${ }^{9}$ since the range of temperatures over which the low-field extremum can be accurately measured experimentally is too small to make such a distinction possible and, more fundamentally, because the position (and amplitude) of the low-field extremum given by the various interpretations assume that $l$ (and $K$ ) are the same over all portions of the Fermi surface which contribute to the low-field extremum. In general, however, both $K$ and $l$ will vary considerably over the Fermi surface, leading to a broadening of the observed $d Z \% d H$ extremum in magnetic

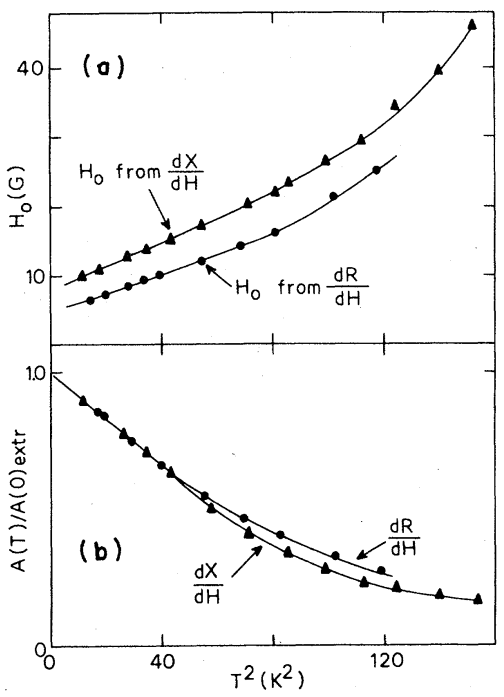

FIG. 3. The position $H_{0}$ and amplitude $A(T)$ of the low-field extremum, shown in Fig. 1, as a function of the square of the temperature $T . A(T) \equiv\left|(d R / d H)_{H_{0}}\right|$ and $A(T) \equiv\left|(d X / d H)_{H_{0}}\right|$ for the extrema observed in the real and the imaginary parts of the surface impedance, respectively. $A(0)_{\text {extr }}$ is the value of $A(T)$ extrapolated to zero temperature. 
field as well as a line shape and amplitude which will be complicated functions of temperature. ${ }^{10}$ Such broadening effects could be expected to be especially large for cylindrical samples where essentially the entire Fermi surface will contribute to the surface impedance, ${ }^{11}$ and it can therefore be expected that an equation such as Eq. (1) will be valid only for small changes in $l$, i.e., for small changes in the temperature. This is illustrated by the fact that a simple temperature dependence for the position of the low-field extremum is, in fact, observed only at low temperatures in molybdenum $\left[H_{0} \simeq T^{2}\right.$, Fig. $\left.3(\mathrm{a})\right]$ where the mean free path exhibits only a weak temperature dependence resulting from residual electron-electron scattering proportional to $T^{2}$ for all major cross sections of the molybdenum Fermi surface. ${ }^{12}$ A similar argument holds for an experimental determination of the temperature dependence of the amplitude of the low-field extremum [Fig. 3(b)]. ${ }^{13}$

In spite of its agreement with all experimental measurements of the low-field extremum, the quantized-skipping-electron interpretation has never received a detailed theoretical treatment in the literature, and it is therefore of considerable interest ${ }^{2}$ to investigate to what extent Eq. (1) can be understood in terms of the well-known Prange-Nee theory for the contribution of skipping electrons to the surface impedance derivative $d Z / d H^{14}$ :

$\frac{d Z}{d H}=$ (const) $\int v \gamma \frac{d}{d h} \sum_{m, n} \frac{(i-\sqrt{3}) \alpha_{m n}^{2}(\beta, h)}{1-h^{2 / 3}\left(\zeta_{m}-\zeta_{n}\right)+(i / \omega \tau)} d A$,

$h \equiv \gamma H=(e / \hbar)\left(v^{3} / 2 K \omega^{3}\right)^{1 / 2} H, \beta \equiv(v / 2 K \omega)^{1 / 2}(1 / \delta)$.

Here $v=v(A)$ is the component of the Fermi velocity parallel to the $\mathrm{rf}$ electric field, $\alpha_{m n}(\beta, h)$ is the matrix element describing the transitions induced between the various quantized-skipping-electron states by the rf electric field, $\zeta_{m}$ and $\zeta_{n}$ are the roots of the corresponding skipping-electron wave functions, $d A$ is the area element of the Fermi surface corresponding to the skipping-electron motion in real space, ${ }^{7}$ and $\tau$ is the relaxation time between effective scattering events for the skipping electrons.

At $\mathrm{GHz}$ frequencies, Eq. (1) rapidly converges yielding a good quantitative description of most of the features experimentally observed in $d Z / d H$ at low magnetic fields. This rapid convergence at $\mathrm{GHz}$ frequencies results from the fact that the relatively small $\mathrm{GHz}$ skin depth effectively limits the summation in Eq. (2) to a small number of terms $\left(\$ 10^{2}-10^{3}\right)$ representing transitions between low-quantum-number skipping-electron trajectories whose depth of penetration into the metal is less than or comparable to the skin depth where the rf electric fields and hence the matrix elements appearing in Eq. (2) are appreciable. At $\mathrm{MHz}$ frequencies the skin depth is much larger and the number of terms required for a similar convergence is too large $\left(\sim 10^{6}-10^{8}\right)$ for direct numerical calculations of all sources of magnetic-field variation in $d Z / d H$ to be practical using Eq. (2). In contrast to $\mathrm{GHz}$ frequencies, however, there will be a large contribution to $d Z / d H$ from transitions between low-quantum-number skipping-electron trajectories which penetrate only a fraction of the $\mathrm{MHz}$ skin depth into the sample. A calculation of this low-quantum-number skipping-electron contribution to the surface impedance has never been carried out using the Prange-Nee theory at $\mathrm{MHz}$ frequencies but such a calculation is of particular interest here since it is precisely the low-quantumnumber skipping-electron trajectories which would be expected to play a major role in determining the position of the low-field extremum according to the quantized-skipping-electron interpretation of Sibbald et $a l .^{1}$ We have therefore carried out such calculations at $\mathrm{MHz}$ frequencies using the same number of low-quantum-number skipping-electron transitions $\left(\sim 10^{2}-10^{3}\right)$ which exhibited excellent convergence at $\mathrm{GHz}$ frequencies. ${ }^{15}$ A broad extremum was found for the theoretically calculated value of $d Z / d H$ at low magnetic fields. However, the convergence of the numerical calculations, especially at higher magnetic fields, is extremely poor, and it must therefore be concluded that the contribution of low-quantum-number skippingelectron trajectories, calculated on the basis of the Prange-Nee theory, cannot explain the lowfield extremum observed experimentally.

In summary, the quantized-skipping-electron interpretation of Sibbald et al. is the only interpretation of the low-field extremum at $\mathrm{MHz}$ frequencies which can account for detailed measurements of the phenomenon in both flat and cylindrical metallic samples and which also agrees with less detailed measurements in other samples. Numerical calculations suggest that a large number of quantized-skipping-electron trajectories contribute to the extremum but a detailed quantitative understanding will require that the Fermi-surface geometry and possibly the nature of electron scattering at the surface be taken into account. In view of the experimental success of the quantized-skippingelectron interpretation and the failure of the Prange-Nee theory to provide a practical quantitative description of the low-field extremum, it is hoped that his work will encourage a more useful and rigorous theoretical formulation of the lowfield surface impedance of metals at $\mathrm{MHz}$ frequencies. 
${ }^{1}$ K. E. Sibbald, A. L. Mears, and J. F. Koch, Phys. Rev. Lett. 27, 14 (1971).

${ }^{2}$ S. Pal and D. S. Falk, Phys. Rev. B 18, 5309 (1978); V. F. Gantmakher, L. A. Falkovskii, and V. S. T soi, Pis'ma Zh. Eksp. Teor. Fiz. Red. 9, 246 (1969). [JETP Lett. 9, 144 (1969)].

${ }^{3}$ V. S. T soi, Zh. Eksp. Teor. Fiz. 64, 214 (1973) [Sov. Phys._JETP 37, 1078 (1973)]; B. E. Meierovich, Zh. Eksp. Teor. Fiz. 59, 276 (1970) [Sov. Phys.-JETP 31, 149 (1971)].

${ }^{4}$ H. D. Drew, Phys. Rev. B 5, 360 (1972).

${ }^{5}$ W. M. MacInnes, B. Collet, P. A. Probst, and R. Huguenin, J. Phys. F 7, 655 (1977); H. H. A. Awater and J. S. Lass, ibid. 3, 1113 (1973).

${ }^{6}$ S. A. Govorkov and V. A. Tulin, Zh. Eksp. Teor. Fiz. 70, 1044 (1976) [Sov. Phys.-JETP 43, 545 (1976)]; V. F. Gantmakher and Yu. V. Sharvin, Zh. Eksp. Teor. Fiz. 39, 512 (1960) [Sov. Phys._JETP 12, 358 (1961)].

${ }^{7}$ The various interpretations of the low-field extremum discussed in this work implicitly assume that the electronic motion is confined to a small portion of the Fermi surface. Qualitatively different phenomena will arise when this condition is not satisfied; e.g., see T. G. Blaney, Philos. Mag. 20, 23 (1969).

${ }^{8} \mathrm{The}$ position of the $d \boldsymbol{Z} / d H$ extremum for a cylinder will be displaced in magnetic field by an amount $\hbar K / e R_{s}$, where $R_{s}$ is the cylinder radius; e.g., see R. E. Doezema, J. F. Koch, and U. Strom, Phys. Rev. 182, 717 (1969).

${ }^{9}$ For example, the same experimental data in copper have been fit equally well to both $H_{0} \sim l^{-3 / 2}$ and $H_{0}$ $\sim l^{-2}$ dependencies, viz., Refs. 1 and 2 . In addition, the semiclassical skipping- and skimming-electron interpretations predict identical frequency and temperature dependences for $H_{0}$, viz., Refs. 2 and 4 .
${ }^{10}$ Such so-called $k_{H}$ broadening effects would also be expected to be more important at low frequencies ( $\omega \tau$ $\lesssim 1$ and $\omega \tau \ll 1$ ) where variations in $K$ (and $l$ ) can affect both the position and the width of the extremum; e.g., see R. A. Gordon and J. B. Frandsen, J. Phys. (Paris) 39 Coll. C6, Supp. No. 8, p. C6-1135 (1978).

${ }^{11} \mathrm{~A}$ detailed analysis of the form of the molybdenum Fermi surface as well as additional measurements in truncated or slightly etched cylinders suggest that the signal in molybdenum originates primarily from small closed regions of the Fermi surface [R. A. Gordon (unpublished)]. The standard assumption that a single localized portion of the Fermi surface is solely responsible for the signal will, however, lead to a more rapid temperature variation in $H_{0}$ over large ranges of temperature; R. A. Gordon, in $P$ roceedings of the Fourteenth International Conference on Low Temperature Physics, edited by M. Krusius and M. Vuorio (North-Holland, Amsterdam, 1975), Vol. 4, p. 333.

${ }^{12}$ V. A. Gasparov, I. F. Voloshin, and L. M. Fisher, Solid State Commun. 29, 43 (1979); M. A. Arutyunyan and V. A. Gasparov, Zh. Eksp. Teor. Fiz. 76, 369 (1979) [Sov. Phys._JETP 49, 188 (1979)].

${ }^{13} \mathrm{~A}$ similar analy sis of the experimental temperature dependence of the amplitude of the low-field extremum has also been carried out for indium; D. G. deGroot, $\mathrm{Ph}$. D. thesis, Vrije University, Amsterdam, 1974 (unpublished).

${ }^{14}$ R. E. Prange and T. W. Nee, Phys. Rev. 168, 779 (1968).

${ }^{15}$ The numerical calculations were carried out in collaboration with J. B. Frandsen using the standard cylindrical Fermi-surface approximation. 